\title{
FUNDAMENTAL LIMITATIONS AND TOPOLOGICAL CONSIDERATIONS FOR FAST DISCHARGE HOMOPOLAR MACHINES
}

M. D. Driga, S. A. Nasar, H. G. Rylander
W. F. Weldon, H. H. Woodson
College of Engineering
University of Texas at Austin
Austin, Texas 78712

Summary

Fusion research experiments require high energy short duration pulses. A homopolar machine, as an inertial energy storage system, offers an attractive source of energy meeting these requirements. The Energy Storage Group at The University of Texas at Austin has investigated the fundamental limitations to the discharge time of homopolar machines of various topological configurations. This paper presents a mathematical model for fast discharge homopolar machines. Based on this model, various machine configurations are analyzed. A new configuration - the spool type machine - is also discussed, criteria for the evaluation of different alternatives are presented, and it is concluded that highly efficient ( $\cong 95 \%)$, high energy ( $\cong$ gigajoules), fast-discharge ( $\cong 5$ to 30 mill iseconds) homopolar inertial energy storage systems are technically feasible. Brief reference is also made to some of the experimental results obtained from the existing laboratory models.

\section{Introduction}

Recently there has been an increasing interest in the fusion research community in the development of homopolar machine systems capable of delivering high energy $\left(\cong 10^{10}\right.$ Joules) - in short duration pulses ( 5 to 30 mill iseconds) $)^{1}$. As fusion experiments increase in size the energy requirements become greater, so the cost of pulse-power supplies becomes an important issue. Furthermore, as trial designs of power producing reactors become refined it is clear that pulse-power supplies are needed not only with reduced cost but also with increased efficiency, high reliability and long life. Homopolar machines, in which the rotor is also a flywheel, become increasingly attractive in meeting these requirements.

The University of Texas at Austin has a program intended to develop a technology which will form the basis for a design procedure for homopolar machines to meet a broad spectrum of performance requirements in terms of stored energy and discharge times. Two years ago the Energy Storage Group at The University of Texas had successfully completed the design, fabrication and testing of a half megajoule homopolar machine. This unit exceeded its design goals by motoring to $6000 \mathrm{rpm}$ in 100 seconds and discharging in 7 seconds with a peak current of over 14,000 Amperes.2 During the last year the Energy Storage Group has designed, fabricated and assembled, a second generation homopolar machine with a storage capacity of five megajoules. 3 This machine produces 42 volts open circuit and has produced a peak current of 0.55 million amperes when short circuited at half rated speed. The rotor stopped in 0.7 seconds in this test. The tests of the machine have shown that the use of conventional technology -solid electrical brushes, room temperature field coils and hydrostatic bearings - leads to reliable, inexpensive and efficient pulse power supplies, attractive for current and future experiments in controlled thermonuclear fusion, for heating and confinement of plasma.
However, a fast discharge homopolar machine represents a step in the development of such machines with design features greatly different when compared to previous designs of homopolar machines. A discharge time between 1 and 100 milliseconds is considered as fast-discharge time. The reversible transfer system for the compression coils in the Reference Theta Pinch Reactor (R.T.P.R.) may require that an energy of the order of 1010 Joules be stored and reversibly transferred with in 10 to 30 milliseconds transfer time and with a cycle efficiency greater than $95 \%$ per cycle.

The aim of this paper is to show that this goal can be successfully achieved by a specially designed system utilizing low-cost high-efficiency homopolar machines. This paper outlines the essential features of the fast discharge homopolar machines, gives an approach for analysis of such machines and defines the fundamental limitations with regard to discharge time and energy-transfer efficiency. The two basic topological alternatives- disk and drum -are compared and performance and design equations are derived. A modified configuration the spool type-is also discussed.

\section{Analytical approach}

\section{Equivalent circuit}

A simple circuit model for a homopolar pulse-power supply connected to a load is an RLC circuit in which the capacitor models the homopolar machine. The expression for the capacitance, $C$, is obtained by equating the kinetic and electric energy $\frac{1}{2} J \Omega^{2}=\frac{1}{2} C V^{2}$, considering that the variables of state which determine the energy, $\Omega$, the angular speed and $V$, the voltage are related by $V=[\Phi /(2 \pi)]_{\Omega}$ for any type of homopolar machine, where $\Phi$ is the total flux (from brush to brush) cut in one revolution.

In a fast discharge machine the picture is complicated by the diffusion of the current (the transient skin effect) in the conductors of the machine, transmission line and load, making the simple RLC model inappropriate. In this case, the current diffuses into the rotor according to the diffusion equation

$$
\frac{\partial^{2} J_{r}}{\partial z^{2}}=\mu \sigma \frac{\partial J_{r}}{\partial t}
$$

The solution to eq. (1) is

$$
J_{n}(z, t)=J_{o} f\left[1-\sum_{n, o d d} \frac{4}{\pi n} \cos \frac{\pi n z}{w} \exp \left(-\frac{n^{2} t}{\tau d}\right)\right](2)
$$

where $J_{o f}=$ final value of the current density, $\tau d=\mu \sigma \mathrm{w}^{2} / \pi^{2}=$ diffusion time-constant, and $w=w i d t h$ of the rotor. The current density, as given by eq. (2), may be integrated over the ent ire thickness of the rotor to obtain the total current, which is also the load current, $i(t)$. Consequently, 
$i(t)=I_{f}\left[1-\sum_{n, \text { odd }} \frac{8}{\pi^{2} n^{2}} \exp \left(-\frac{n^{2} t}{\tau d}\right)\right]$

where $I_{f}$, the final current, is limited by $R_{m}$, the total resistance of the circuit; that is $I_{f}=V / R_{m}$.

In order to represent the current diffusion, as given eq. (2), by an equivalent circuit, consider the network shown below

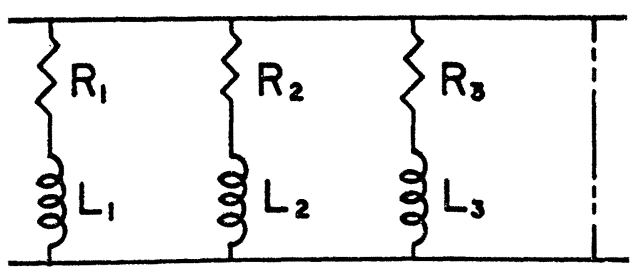

Fig. 1 - Equivalent circuit for current diffusion.

for which the current in the nth branch is

$i_{n}(t)=\frac{V}{R_{n}}\left[1-\exp \left(-R_{n} t / L_{n}\right)\right]$

and the total current is simply

$i(t)=\sum_{n, \text { odd }} \frac{V}{R_{n}}\left[1-\exp \left(-R_{n} t / L_{n}\right)\right]$

Eqs. (3) and (5) are equivalent if $R_{n}=\pi^{2} n^{2} / 8 R_{m}$ and $L_{n}=$ $\mu \sigma w^{2} R_{m} / 8=L_{m}$. With these parameters, the diffusion phenomenon may be modelled by the equivalent circuit ${ }^{4}, 5$ in Fig. 1.

For the two topological alternatives of homopolar machine -the disk and the drum - the transmission lines are cocxial cylinders or parallel plates - the values for the elements of the equivalent networks are given in the appendix.

The equivalent circuit modelling a fast discharge system becomes as shown in Fig. 2.

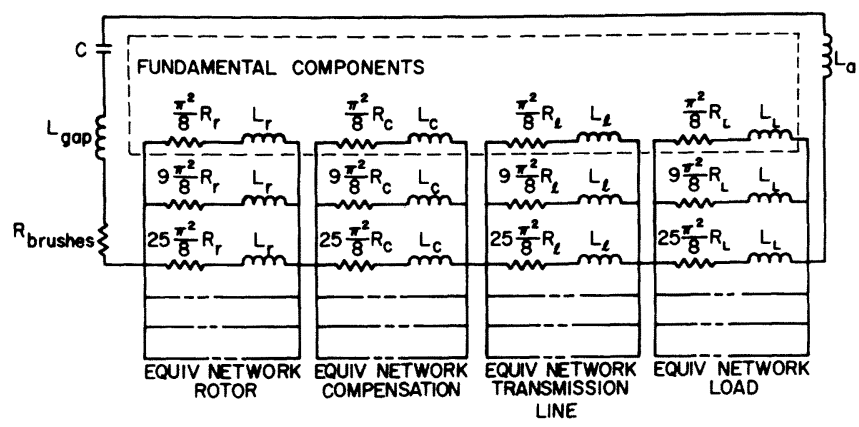

Fig. 2 - Equivalent circuit of homopolar fast-discharge system.

This circuit can be solved on a computer without diffculty, using the number of branches needed for the desired accuracy. However, in this paper, for finding the basic limitations, only the fundamental term of the fast coverging Fourier series is considered. Admittedly, in doing so some accuracy is lost, but the overall results for preliminary estimates can be quickly obtained. This results in a simple RLC circuit, shown in Fig. 3, which, for efficiency considerations, is designed to be highly underdamped.

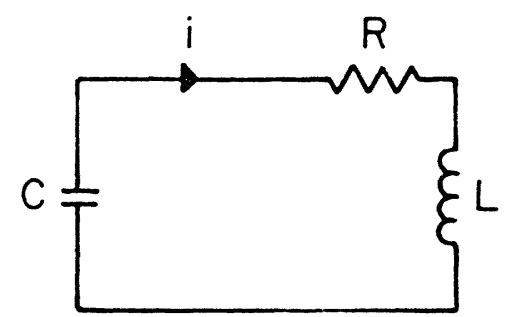

Fig. 3 - An approximate equivalent circuit (RLC are total values).
Having obtained the equivalent circuit, the electrical characteristics of the fast-discharge homopolar machine system can be obtained. The three important quantities - discharge time, efficiency of discharge, and losses in bearings - which are significant in assessing the performance of the machine are now considered.

Discharge time

The current variation in a circuit such as the one shown in Fig. 3 is given by:

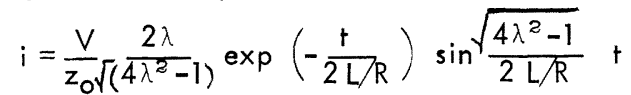

where $z_{0}=\sqrt{L / C}$ and $\lambda=z_{0} / R$. The kinetic energy stored in the rotor is transferred to the load when $i$ reaches its maximum value; that is after,

$$
\tau_{e}=\frac{\pi}{2 \sqrt{\left(4 \lambda^{2}-1\right)}} \approx \frac{\pi}{2} \sqrt{L C}
$$

because, for efficiency $\geq 95 \%, \lambda \geq 30$. It should be noted that the delay because of the current diffusion is taken into account approximately by increasing the values of the inductances for different elements of the machine, transmission line and the load. Eq. (7) may also be written as:

$$
\tau_{e}=\frac{\pi}{2} \sqrt{\left(L_{i}+L_{e}\right) C}=\frac{\pi}{2} \sqrt{L_{i} C} \sqrt{1+L_{e} / L_{i}}=n_{s h}{ }_{s}^{(8)}
$$

where $n=\sqrt{1+L_{e} / L_{i}}$ is a coefficient of load compatibility and $\tau_{s h}=\sqrt{L_{i} C}$ is the short-circuit discharge time.

\section{Efficiency of discharge}

The mechanical losses during a discharge being negligible as compared to electrical losses, the energy efficiency during discharge is defined as:

$$
\begin{aligned}
\eta=1 & -\frac{\text { energy lost in resistance, during } \tau_{e}}{\text { energy stored in the rotor at } \Omega_{0}} \\
& -\frac{\text { energy which remained in } L_{i} \text { during } \tau_{e}}{\text { energy stored in the rotor at } \Omega_{0}}
\end{aligned}
$$

It may be shown that ${ }^{7}$

$$
\eta=\left(1-\frac{1}{n^{2}}\right) \exp \left(-\frac{\pi \alpha^{2}}{2 n \lambda_{s h}}\right)
$$


where $\lambda_{s h}=\frac{1}{R_{i}} \sqrt{L_{i} / C}$ is given for the shortcircuited machine and $\alpha=\sqrt{1+R_{\ell} / R_{i}}$ is a coefficient of compatibility.

\section{Losses in bearings}

The sequence of typicaloperation cycle is shown in Fig. 4. When the kinetic energy stored in the rotor is rapidly discharged into the load and when, after the experiment having been performed, the remaining energy is returned back to the rotor which then builds up speed $\left|\Omega^{\prime}\right|<\left|\Omega_{0}\right|$ the mechanical losses can be neglected as compared to the electrical losses. However, in the intervals $\tau_{2}$ and $\tau_{4}$ (Fig. 4) these losses can play an important role. In the interval $\tau_{2}$ the machine will oscillate, until the energy stored in the internal inductance of the machine and transmission line is dissipated.

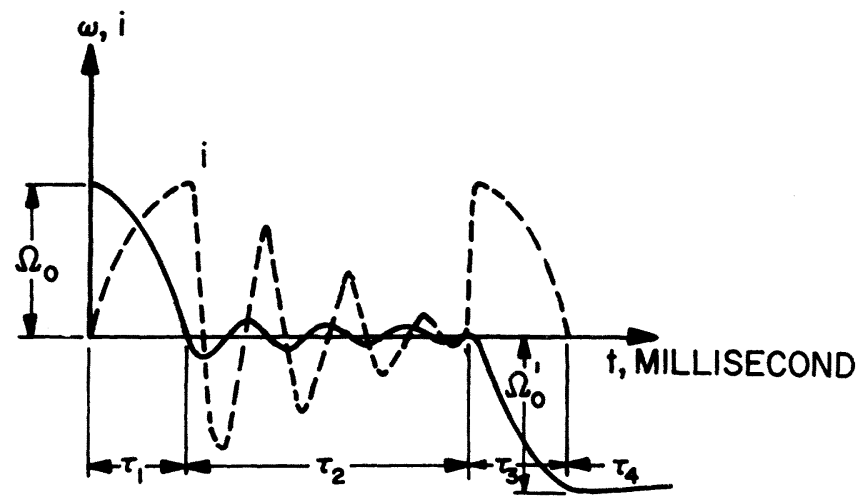

(a)

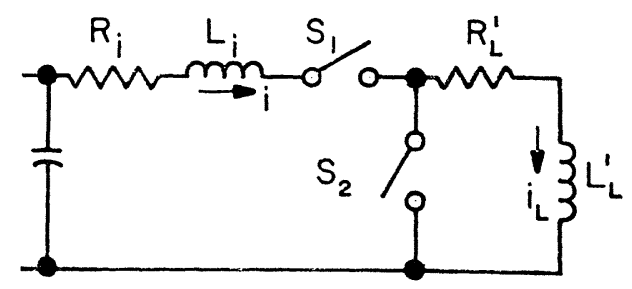

(b)

Fig. 4 (a) Sequence of operation of a fast-discharge machine (b) Equivalent circuit (with switches) $\tau_{1}=$ discharge into load $\left(S_{1}\right.$ closed, $S_{2}$ open); $\tau_{2}=$ oscillation of shortcircuited machine $\left(S_{1}\right.$ closed, $S_{2}$ closed); $\tau_{2}=$ energy returned to the rotor $\left(S_{1}\right.$ closed, $S_{2}$ open $) ; \tau_{4}=$ coasting $\left(S_{1}\right.$ open, $S_{2}$ open).

The interval $\tau_{4}$ - the coasting interval - is a long one, separating two cycles of operation. The brushes are lifted and the losses in the bearings proportional to the square of the peripheral speed ${ }^{7}$ are the principal losses in the machine. They are taken into consideration by a conductance $G_{B}$ in parallel with the capacitance.
The losses in bearings $=G_{B} V^{2}=K \Omega^{2}$, where $K=$ bearing design constant and $G_{B}=4 \pi^{2} K / \Phi^{2}$.

For the interval $\tau_{2}$ the equivalent circuit is shown in Fig. 5 , where $R_{i}$ and $L_{i}$ represent respectively the values for the inductance of the machine and of the transmission line, with the correction (the first harmonic) for the transient skin effect.

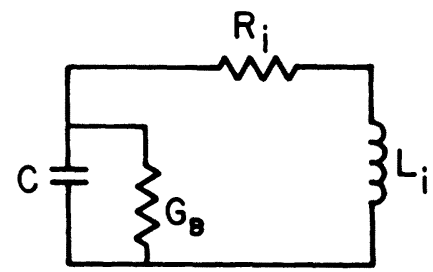

Fig. 5 - Equivalent circuit during $\tau_{2}$.

In this case, the characteristic equation

$$
p^{2}+\left(\frac{R_{i}}{L_{i}}+\frac{G_{B}}{C}\right) p+\left(\frac{R_{i}}{L_{i}} \frac{G_{B}}{C}+\frac{1}{L_{i} C}\right)=0
$$

$$
\text { has the solution } p_{1,2}=-\left(\frac{R_{i}}{2 L_{i}}+\frac{G_{B}}{2 C}\right) \pm i \sqrt{\frac{1}{L_{i} C}-\left(\frac{R_{i}}{2 L_{i}}-\frac{G_{B}}{2 C}\right)^{2}}
$$

The effect of the losses in the bearings is to increase the resulting damping constant as may be seen in the interval $\tau_{2}$ (Fig. 4). These losses increase the angular frequency. However, this effect is predominately due to the sharp drop of the inductance of the circuit rather than the increase in bearing losses. In the interval $\tau_{4}$ (coasting) the equivalent circuit is shown in Fig. 6, the losses are $G_{B} V^{2}$, and the speed (and consequently the voltage at a constant excitation) falls off exponentially, with a time constant $\tau_{C}=C / G_{B}$.

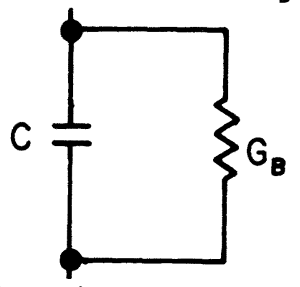

Fig. 6 - Equivalent circuit during $\tau_{4}$.

\section{Topological considerations}

Two previous reports ${ }^{6}, 8$ have shown that, ideally, the basic topological configurations- the disk and the drum (Fig. 7) (and also different intermediate topological alternatives) are equivalent. For homopolar machine, with ferromagnetic rotor and yoke it was found ${ }^{8}$ that the drum and disk configurations are equivalent if evaluated by the specific power index (power per unit volume).

For energy storage and discharge applications, the rapidity at which the energy stored can be transferred to the load and the efficiency of this transfer are the two important parameters. In this case the capacitance of the machine can be expressed as $C=4 \pi^{2} \mathrm{~J} / \Phi^{2}$ and the discharge time 


$$
\tau_{D}=\pi^{2} \sqrt{L J / \Phi}=K_{D} \sqrt{J} / \Phi, \text { where } K_{D}=\pi^{2} \sqrt{L}
$$

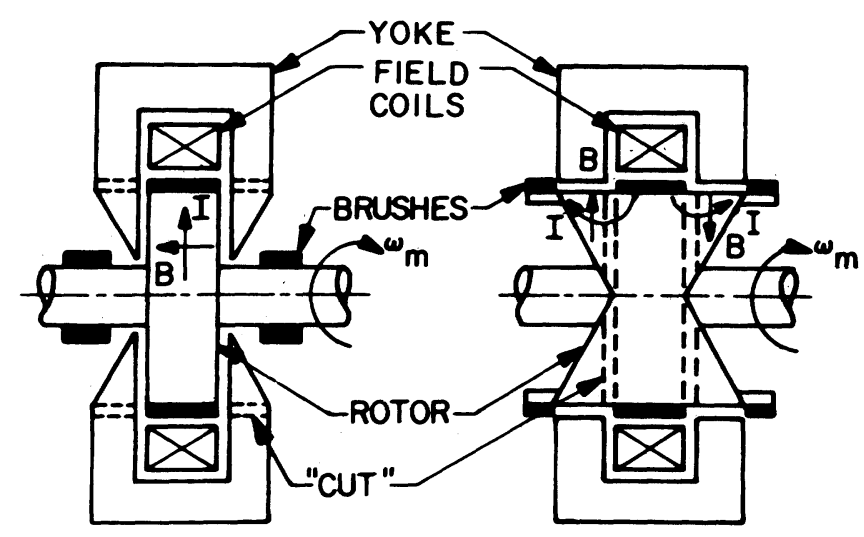

(a) DISK TYPE

(b) DRUM TYPE

Fig. 7 - Topological transformation of a disk machine into a drum machine.

The ratio of the moments of inertia ${ }^{8}$

$$
\frac{\mathrm{J} \text { disk }}{\mathrm{J} \text { drum }}=\frac{w}{w+0.8 r_{0}}
$$

shows that the disk machine, when used in pulse-power application is qualitatively superior to the drum machine (equivalent to the disk) having the same specific power index. The reason is that for the same flux, a part of the magnetic circuit (Fig. 7) is transferred to the rotor for the drum machine, thereby increasing its moment of inertia. For the fast discharge homopolar machines it is preferable to use a nonferromagnetic material (for which $\mu=\mu_{0}$ ) for the rotor because of the diffusion time constant considerations, as derived earlier.

Because $\operatorname{div} \bar{B}=0$ must hold everywhere the total flux inducing the voltage in the rotor is the same for the two topologically equivalent rotors of identical external radius and length and identical field coils (Fig. 8), assuming the same reluctance in the two cases.

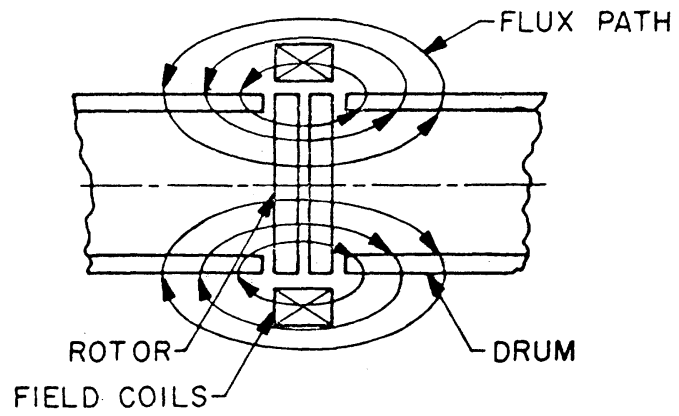

Fig. 8 Flux lines in a homopolar disk or drum machine.
The use of simplified formulas such as $B l u=V$ leads to erroneous conclusions in this case, owing to the nonuniform distribution of magnetic flux density. The modified counterrotating disk machine (or the spool machine) has a smaller moment of inertia and consequently a smaller discharge time (Fig. 9)
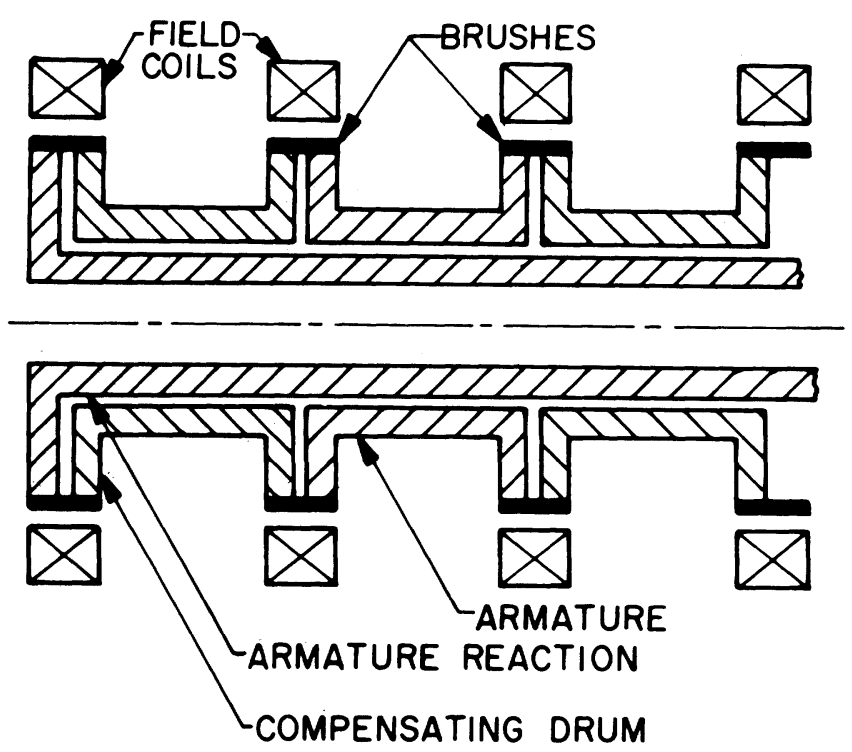

Fig. 9 - Counter-rotating spool machine.

than the drum machine (Fig. 10). A comparison between these two alternatives yields the following conclusions:

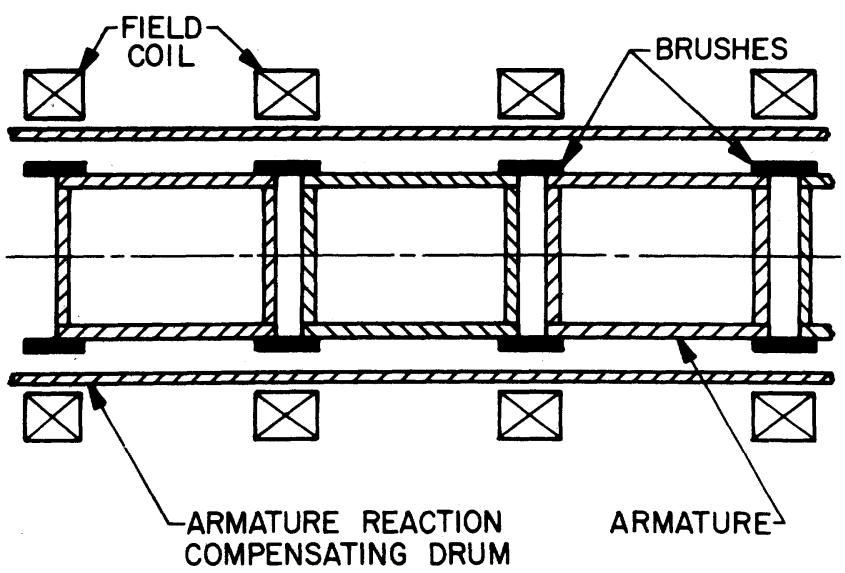

Fig. 10 - Multiple coil multi-drum machine.

The spool machine (Fig. 9) will give a higher voltage and faster discharge than the drum machine (Fig. 10), because in the drum machine the rotor has to be farther from the field coil-at a distance equal to the width of the compensating cylinder. Because of lower rotational stress, the spool can be rotated at a higher angular speed, and thereby increasing the voltage and at the same time, partially compensating for the fact that the spool stores less energy than the drum. The stresses in the compensating cylinder for the drum machine, located in the highest field density region, are much higher, than in the compensating (inner) cylinder of the disk machine. The total angular momentum of two thin counterrotating spools in close proximity is nearly zero. The almost perfect local compensation of the angular momentum substantially reduces 
the stresses transmitted to the stator. Supplementary problems appear for the drum machine due to the interconnections between the drums, brushes, and compensating cylinder at a high voltage $(\cong 10,000 \mathrm{v})$.

\section{Short circuit parameters and} fundamental limitations of the machine

A fast discharge machine is characterized by two basic parameters: the time of discharge and the efficiency of discharge. The efficiency is related to the parameter $\lambda$, which was assumed to be over 30 for a $95 \%$ efficiency. In this case

$$
\tau_{e}=\frac{\pi}{2} \sqrt{L C}=\frac{\pi}{2} \sqrt{L_{i} C} \sqrt{1+\frac{L_{L}}{L_{i}}}=\tau_{s h} n
$$

and

$$
\lambda=\frac{1}{R} \sqrt{\frac{L}{C}}=\frac{n}{R_{i}} \sqrt{\frac{L_{i}}{C}} \sqrt{\left(1+\frac{R_{l}}{R_{i}}\right)^{2}}=\lambda_{s h} \frac{n}{\alpha^{2}}
$$

The "fast discharge" characteristic is a property of the system - machine, transmission line, and the load - and not a property of the machine alone. For this reason an attempt to analyze the machine alone, to find the condition for a fast discharge, is incorrect. However, the capacitance of the system is concentrated in the machine only and the system can be characterized by relating the shortcircuit parameters of the machine to the load by the coefficients of compatibility $n$ and $\alpha$.

The discharge time of the shortcircuited machine represents the shortest time in which that machine can be "discharged" and permits the evaluation of "fundamental limitations". The following results are summarized from Ref. 6:

For the drum machine

$$
\begin{aligned}
& \tau_{\text {sh }}^{\prime}=\frac{\pi^{2} w r_{0}^{2}}{\Phi} \sqrt{\frac{\mu_{o} \rho m}{2}} \sqrt{\bar{J} \text { drum } \bar{L} \text { drum }} \\
& \lambda_{\text {sh }}^{\prime}=\frac{4 \sigma w}{\pi^{3}} \frac{\Phi}{r_{0}^{2} \bar{R} \text { drum }} \sqrt{\frac{\mu_{0}}{2 p_{m}}} \sqrt{\frac{\bar{I} \text { drum }}{\bar{J} \text { drum }}}
\end{aligned}
$$

For the spool machine

$$
\begin{aligned}
& \tau_{\text {sh }}^{\prime \prime}=\frac{\pi^{2} w_{0}^{2}}{\Phi} \sqrt{\frac{\mu_{0} \rho m}{2}} \sqrt{\bar{J} \text { spool } \bar{L} \text { spool }} \\
& \lambda_{\text {sh }}^{\prime \prime}=\frac{4 \sigma w}{\pi^{3}} \frac{\sigma}{r_{0}{ }^{2} \bar{R} s p o o l} \sqrt{\frac{\mu_{0}}{2 \rho_{m}}} \sqrt{\frac{\bar{L} \text { spool }}{J_{s p o o l}}}
\end{aligned}
$$

where $\bar{L}, \bar{J}$, and $\bar{R}$ are nondimensional coefficients and respectively characterize the inductance, moment of inertia and resistance of one topological alternative or the other.

It is important to note that:(a) The discharge time and the efficiency of the machine do not depend on the energy stored in the machine, that is, on the gngular speed of the rotor; and (b) The transient skin effect for all the elements of the system is transformed from a field problem to a circuit problem.

\section{A design criterion}

In the design of classical electrical machines certain coefficients of "quality" are introduced. For a fast discharge machine such a coefficient is $G=\lambda_{s h} / \tau_{s h}$. For the two configurations under consideration:

$$
\begin{aligned}
& G \text { drum }=\frac{4}{\pi^{5}} \frac{\Phi^{2}}{\rho_{e} \rho_{m}} \cdot \frac{1}{r_{0}^{4} \bar{J} \text { drum }} \cdot \frac{1}{\bar{R} \text { drum }} \\
& G \text { spool }=\frac{4}{\pi^{5}} \cdot \frac{\Phi^{2}}{\rho_{e} \rho_{m}} \frac{1}{r_{o}^{4} \bar{J} \text { disk }} \cdot \frac{1}{\bar{R} \text { disk }}
\end{aligned}
$$

$G$ has a maximum not for the maximum flux density, which, is a variable along the rotor, but for its integral over the lateral area of the rotor. The product $p^{\rho}$ of the density of the material and electrical resistivity must be as small as possible. Aluminum has the smallest value among the known suitable materials. Also, from mechanical considerations aluminum is very suitable as rotor material for fast discharge machines. The topological configuration of the rotor, its influence on the discharge time and on the efficiency of the machine, is taken into consideration by the product of the nondimensional coefficients ( $1 / \bar{J}$ spool) $(1 / \bar{R}$ spool) or ( $I / \bar{J}$ drum $)(T / \bar{R}$ drum).

Fast discharge machine characteristics

The criteria for evaluating the fast discharge machines are the discharge time and the efficiency of the discharge. Curves (Fig. 11) are plotted for the two configurations, for aluminum rotors of different thickness. The load is assumed to be a coil having an inductance of $10^{-6} \mathrm{H}$ and a resistance of $10^{-7} \Omega$.

The curves show that a spool machine on load can achieve smaller discharge times than the equivalent drum, at a high efficiency, because the capacitance of the spool is smaller as compared to the equivalent drum. The capacitance values for the shortcircuit parameters show the main drawback for the spool machine - a higher internal inductance leading to higher values for $\tau$. However, only the performance of the machine connected to the load is of interest, and the inductance of the machine is negligible as compared to the inductance of the load. Because of the smaller capacitance, the energy stored in the spool is smaller than in the equivalent drum in the ratio: $W$ spool $/ W$ drum $=\bar{J}$ spool $/ J$ drum for the same angular speed $\Omega_{0}$.

If the number of machines connected in series is modified to achieve the same stored energy, the discharge time of the system powered by the spoolmachine will be smaller, in the ratio: $\tau$ spool $/ \tau$ drum $=\bar{J}$ spool $/ \bar{J}$ drum. However, mechanical considerations show that the spoolcan be rotated faster than the equivalent drum, partially offsetting the difference in the stored energy.

\section{Conclusions}

The paper shows that in homopolar inertial energy-storage systems, discharge times in a few mil liseconds range are technically feasible. Also, whereas ideally the two topological alternatives are equivalent, the disk machine (or spool) can be achieve smaller discharge times because of its lower capacitance. An analytical approach to a fast discharge system with homopolar machines is possible by modelling the transient skin effect for different elements of the system by parallel RL networks. Measu:ements of shortcircuit parameters $\tau_{\text {sh }}$ and $\lambda_{\text {sh }}$ can be performed at low angular speeds. The results characterize the machine operation at different speeds and, through the coefficients of compatibility, at different loads. 


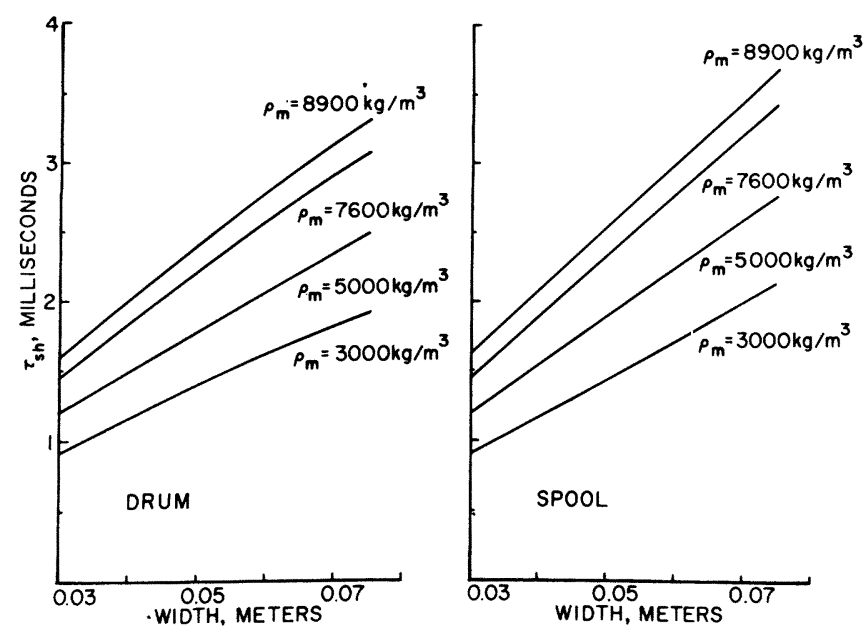

(a)

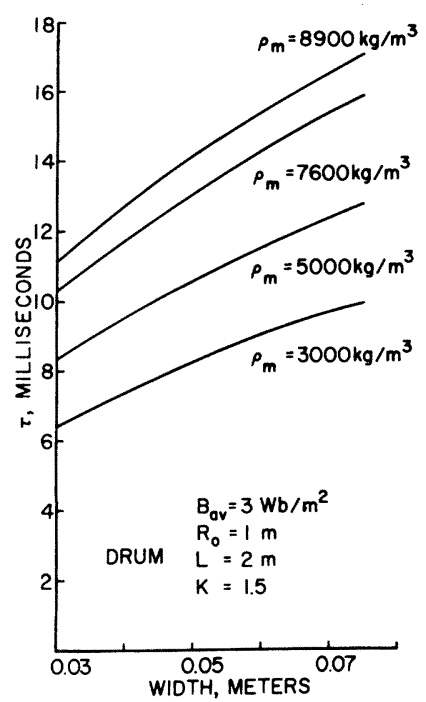

(b)
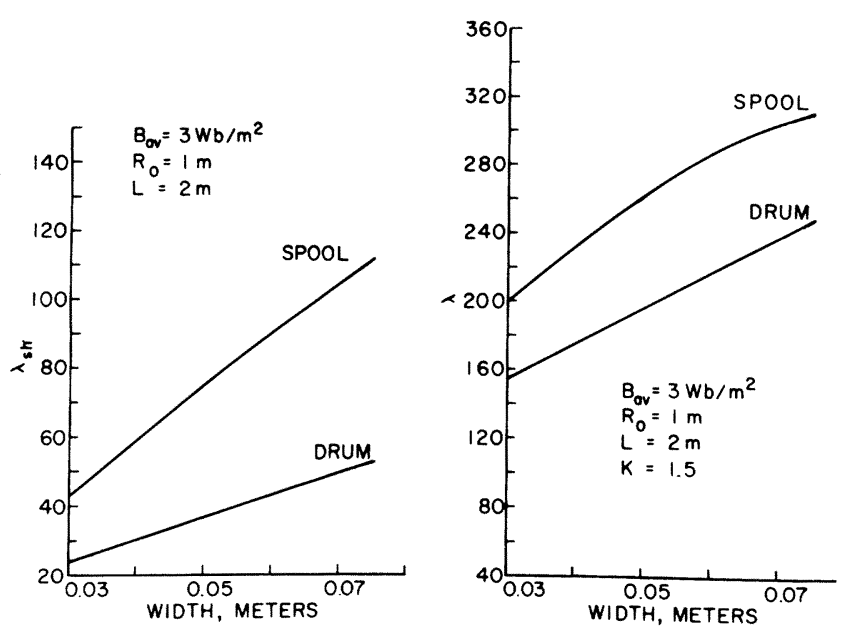

(c)

Fig. 11(a) - Short-circuit discharge time for drum and spool machines. (b) On-load discharge time for drum and spool machines. (c) Effect of machine width on short-circuit and on-load $\lambda$ - parameters.

\section{Aknowledgements}

The work reportedhere was supported by Energy Research and Development Administration (formerly the U. S. Atomic Energy Commission), the Electric Power Research Institute and the Texas Atomic Energy Research Foundation

\section{Appendix}

The various $R, L, C$ components modelling the fast discharge system including current penetration wers obtained in ${ }^{6}$ :

\section{Resistances}

a) Drum machine

$$
\begin{aligned}
& R_{t}^{\prime}=\frac{\pi^{2}}{8} \cdot \frac{\rho_{e}}{\pi w}\left[\frac{l}{r_{0}}+\frac{8}{\pi^{2}}\left(\frac{6.45 \times 10^{-8} w}{\rho_{e} b_{0}}\right)\right] \\
& =\bar{R} \operatorname{drum}\left[\frac{\rho_{e} e^{\pi}}{8 w}\right]
\end{aligned}
$$

b) Spool machine

$$
\begin{aligned}
R_{t}^{\prime \prime}=\frac{\pi^{2}}{8} & \frac{\rho_{e}}{\pi w}\left[\operatorname{lnk}+\frac{l}{r_{0}}+\frac{8}{\pi^{2}}\left(\frac{6.45 \times 10^{-8} w}{\rho_{e} b_{0}}\right)\right] \\
& =\bar{R} \text { spool } \frac{\pi}{8 \sigma w} \\
\text { where } \bar{R} \text { spool } & =\operatorname{lnk}+\frac{l}{r_{0}}+\frac{8}{\pi^{2}}\left(\frac{6.45 \times 10^{-8} w}{b r_{0} \rho_{e}}\right)
\end{aligned}
$$

Inductances

a) Drum machine

$$
\begin{aligned}
& L_{f}^{\prime}=\frac{\mu_{0} w}{2 \pi}\left[\frac{l}{r_{0}}\left(1+\frac{\delta}{w}\right)\right]=\bar{L} \text { drum }\left(\frac{\mu_{0} w}{2 \pi}\right) \\
& \text { where }\left[\text { drum }=\frac{l}{r_{0}}\left(1+\frac{\delta}{w}\right)\right.
\end{aligned}
$$

b) Spool machine

$$
\begin{aligned}
& \begin{aligned}
L_{t}^{\prime \prime} & =\frac{\mu_{0} w}{2 \pi}\left[\left(1+\frac{\delta}{w}\right) \operatorname{lnk}+\frac{\delta^{\prime}}{w}\left(\frac{l-w}{r_{0} / k-k w}\right)+\frac{l}{2 r_{0}}\left(k^{2}+k^{12}\right)\right] \\
& =\left[\operatorname{spool}\left(\frac{\mu_{0} w}{2 \pi}\right)\right.
\end{aligned} \\
& \text { where }\left[\text { spool }=\left(1+\frac{\delta}{w}\right) \operatorname{lnk}+\frac{\delta^{\prime}}{w}\left(\frac{l-w}{r_{0} / k-k w}\right)+\left(k^{2}+k^{12}\right) \frac{l}{2 r_{0}}\right.
\end{aligned}
$$

\section{Capacitances}

a) Drum machine

$$
\begin{aligned}
& \begin{aligned}
C_{\text {drum }} & =\frac{4 \pi^{3} \rho_{m} w r_{0}^{4}}{\dot{\psi}^{2}}\left[1+\frac{2 l}{r_{0}}\left(1-\frac{1.5 w}{r_{0}}\right)\right] \\
& =J \text { drum } \frac{4 \pi^{3} \rho_{m} w r_{0}^{4}}{\Phi^{2}}
\end{aligned} \\
& \text { where } J \text { drum }=1+\frac{2 l}{r_{0}}\left(1-\frac{1.5 w}{r_{0}}\right)
\end{aligned}
$$


b) Spool machine

$$
\begin{aligned}
& C_{\text {spool }}= \frac{4 \pi^{3} \rho_{m} w r_{0}^{4}}{\Phi^{2}}\left\{1-\frac{1}{k^{4}}+\frac{l}{2 w}\left[\frac{1}{k^{4}}-\left(\frac{1}{k}-\frac{k w}{r_{0}}\right)\right]\right\} \\
&= J \text { spool } \frac{4 \pi^{3} \rho_{m} w r_{0}^{4}}{\Phi^{2}} \\
& J_{\text {spool }}=1-\frac{1}{k^{4}}+\frac{l}{2 w}\left[\frac{1}{k^{4}}-\left(\frac{1}{k}-\frac{k w}{r_{0}}\right)^{4}\right] \\
& \text { References }
\end{aligned}
$$

1. F. L. Ribe, Fusion reactor systems, Reviews of Modern Physics, vol. 47, no. 1, Jan. 1975.

2. H. G. Rylander, H. H. Woodson, E. R. Becker, and R. E. Rowberg, Homopolar motor-generator de isgns, for inertial energy storage. Proceedings of Symp. on Technology of Controlled Thermonuclear Fusion Experiments, Austin, Texas No. 20-22, 1972.

3. W. F. Weldon, M. D. Driga, H. H. Woodson, H. G. Rylander, The design fabrication and testing of a five me- gajoule homopolar motor generator, Proceedings of the International Conference on Energy Storage, Compression and Switching, Torino (Italy) Nov. 5-7, 1974.

4. P. Silvester, Modal network theory of skin effect in flat conductors, Proc. IEEE, vol. 54, pp. 1147-1151, 1966.

5. C. I. Mocanu, The equivalent schemes of cylindrical conductors at transient skin effect, IEEE Trans. PAS-72, May-June, pp. 844-852.

6. M. D. Driga, S. A. Nasar, H. G. Rylander, W. F. Wot don, and H. H. Woodson, Fast discharge acyclic machines, Internal report, Energy Storage Group, Univ. of Texas, April 1975.

7. S. Pinkus, Theory of Hydrodynamic Lubrication, McGraw Hill, 1961, pp. 49-58.

8. M. D. Driga, S. A. Nasar, H. G. Rylander, W. F. Wet don, and H. H. Woodson, A topological approach to acyclic machines, Internal report, Energy Storage Group University of Texas, March 1975. 